\title{
Perfil de Complicaciones Tempranas en la Atención del Paciente Traumatizado
}

\author{
${ }^{1}$ Ezequiel Monteverde, ${ }^{2}$ Laura Bosque, ${ }^{3}$ Betina Lartigue, ${ }^{4}$ Emilio Maciá, ${ }^{5}$ Cristian Barbaro \\ ${ }^{6}$ Claudio Ortiz, ${ }^{7}$ Enrique Ginzburg, ${ }^{8}$ Jorge Neira
}

\section{RESUMEN}

Introducción y objetivos: Las estrategias de mejora de la calidad de atención (MCA) ponen foco en las complicaciones prevenibles surgidas durante la internación de pacientes traumatizados, considerando que su prevención mejora los resultados asistenciales.

Materiales y métodos: Se llevó a cabo un estudio retrospectivo, observacional usando datos deidentificados de pacientes ingresados sucesivamente al Registro de la Fundación Trauma en ocho hospitales de Buenos Aires, Argentina. Estos hechos fueron incluidos cuando cumplieran criterios de inclusión (internados $>23$ hs o fallecidos/egresados sin alta médica $<23 \mathrm{hs}$ ). Las complicaciones fueron estudiadas de acuerdo a 163 definiciones preestablecidas, incluyendo complicaciones generales $(C G)$ y relacionadas con procedimientos $(C P)$. Además fueron clasificadas como tempranas (CT) $(<72$ h) y tardías (> $72 \mathrm{~h}$ ).

Resultados: Se reportaron 4521 complicaciones en 1883 pacientes (22\%), mediana 1, RIC 1-3, p/paciente, distribuyéndose en 3586 CG (79\%) y 935 CP (21\%). Las CG se concentraron más en las primeras 72 hs: CG vs CP: 69 vs 49\% ( $p<0,001)$. Las CG más frecuentes fueron paro cardiorrespiratorio $(8,6 \%)$, neumonía asociada al ventilador $(5,6 \%)$, delirio $(4,7 \%)$ e hipertensión endocraneana (4\%); las CP más comunes: inconvenientes en disposición de insumos (64\%) y la derivación de pacientes (14\%). La mortalidad global fue $5.5 \%$; en el grupo de CT fue $29.4 \%$, claramente superior al resto (1.5\%). EI OR para mortalidad en el grupo CT fue 5 veces el de complicaciones totales. Luego de eliminar interacciones y variables no significativamente asociadas al resultado, el modelo de regresión integrado por edad $<20$ años, ISS $>8$, shock al ingreso y GCS < 9 se asoció a un OR de 11.5 (IC95\% 8.8-15.0) de presentar CT (área bajo ROC 0,75).

Conclusiones: En una muestra de pacientes traumatizados internados obtenida de cuatro años en ocho hospitales argentinos, se observó un perfil específico de paciente con mayor riesgo de sufrir CT, lo que puede ayudar en las estrategias de MCA.

\footnotetext{
1-5,7,8 Fundacion Trauma, Ciudad Autónoma de Buenos Aires, Argentina

${ }^{6}$ Ministry of Health, Buenos Aires Province, Argentina

Corresponding Author: Jorge Neira, Fundacion Trauma Ciudad Autónoma de Buenos Aires, Argentina, e-mail: jneira@fundaciontrauma.org.ar
}

Palabras claves: Complicaciones, Mejora de la calidad, Mortalidad, Registro de trauma.

How to cite this article: Monteverde $E$, Bosque L, Lartigue B, Maciá E, Barbaro C, Ortiz C, Ginzburg E, Neira J. Perfil de Complicaciones Tempranas en la Atención del Paciente Traumatizado. Panam J Trauma Crit Care Emerg Surg 2015;4(3):165-171.

\section{Source of support: Nil}

Conflict of interest: None

\section{ABSTRACT}

Introduction: Trauma quality improvement (TQI) strategies put their focus on complications during patient hospitalization, considering that its prevention may improve patient outcomes.

Materials and methods: We carried out a retrospective observational research using de-identified data of prospectively recorded patients in the Trauma Registry hosted by Fundacion Trauma in eight hospitals located in Buenos Aires, Argentina. These cases were included if fulfilled inclusion criteria (hospitalized $>23$ hours or transferred out, elopement or died in the first 23 hours). Complications were studied according to 163 pre-established definitions, including general (GC) and procedure-related complications (PRC), which were also classified as early (EC; $<72$ hours) and late complications ( $L C$; $>72$ hours).

Results: Around 4521 complications were voluntarily reported in 1883 patients (22\%), accounting for $1(1-3)$ complication per patient (median, IQR). $79 \%(n=3586)$ were GC and $21 \%(n=$ 935) were PRC. General complications were more frequent in the first 72 hours (GC vs PRC: 69 vs 49\%, p < 0.001).

The most common GC were cardiorespiratory arrest (8.6\%), ventilator associated pneumonia (5.6\%), delirium $(4.7 \%)$ and intracranial hypertension (4\%). The most common PRC were administrative: either in device provision (64\%) or in the transfer-out procedure (14\%). Global mortality was 5.5\%. Mortality in patients with EC was $29.4 \%$ as compared to $1.5 \%$ in those without EC. Odds ratio (OR) for mortality in EC group was increased five times that of LC group. After eliminating interactions and variables not significantly associated to outcome, a regression model integrated by age $<20$, an injury severity score $>8$, shock at admission and Glasgow coma scale $<9$ was associated to an OR of 11.5 (95\% Cl 8.8-15.0) of having an EC (area under ROC curve 0.75).

Conclusion: In a sample of hospitalized injured patients from a 4-year period in eight Argentinian hospitals, we identified a specific profile with a higher risk for suffering EC. The detection

Paper presented in General Research Competition in PTS Congress, Panama City, Panama, Nov 2014. 
of patients with this profile early at admission may reveal useful for TQI programs.

Keywords: Complication, Mortality, Quality improvement, Trauma registry.

\section{INTRODUCCIÓN Y OBJETIVOS}

El trauma es la principal causa de muerte entre 1 y 45 años en la Argentina, ${ }^{1}$ cifra que coincide con las reportadas por la OMS. En Argentina, el trauma representa el 7\% de la mortalidad global. Si bien se trata de un problema de alcance global, en los países desarrollados se ha reportado en las últimas décadas una caída en la mortalidad por esta causa, principalmente como consecuencia de la organización de las instituciones sanitarias dentro de sistemas de trauma. ${ }^{2-5}$

El aumento de la superviencia de los pacientes traumatizados, por otro lado, ha puesto de manifiesto las complicaciones del tratamiento de estos pacientes. Las complicaciones en el tratamiento de los pacientes traumatizados son causa de mayor morbimortalidad, estadía hospitalaria, secuelas e incremento de costos. ${ }^{6,7}$ Las estrategias de mejora de la calidad de atención (MCA) ponen foco en las complicaciones prevenibles surgidas durante la internación de pacientes traumatizados, considerando que su prevención mejora los resultados asistenciales. $^{8}$

El propósito de la Fundación Trauma es brindar al Sistema de Salud un registro de trauma (RT) que pueda servir de base para la organización de un sistema de trauma. El RT inició su actividad en enero de 2010 en un hospital, incorporando progresivamente el resto de las instituciones (la última, en mayo de 2012).

El objetivo de este trabajo fue evaluar la incidencia y tipo de complicaciones que ocurren en pacientes traumatizados y determinar un perfil de riesgo para la ocurrencia de complicaciones tempranas que ayude a elaborar estrategias preventivas en un subgrupo adecuadamente definido.

\section{MATERIALES Y MÉTODOS}

Se llevó a cabo un estudio retrospectivo, observacional usando datos deidentificados de pacientes ingresados sucesivamente al Registro de Trauma (RT) de la Fundación Trauma en ocho hospitales de la provincia de Buenos Aires, Argentina. El RT incorpora pacientes que cumplan con la definición de trauma de la OMS y permanezcan más de 23 horas en los hospitales, excepto que fallezcan, sean derivados o egresen sin alta médica antes de ese tiempo, caso en el cual son también registrados. El RT codifica a través de AIS, CIE, GOS, RTS, ISS, NISS y TRISS.
En el período 01/2010-07/2014 se registraron 10772 hechos en ocho hospitales, de los cuales 8382 estaban cerrados se incluyeron en el análisis. Los datos obtenidos del RT fueron: edad, género, mecanismo lesional, procedimientos, estadía hospitalaria, complicaciones y mortalidad dentro del hospital. Las complicaciones fueron estudiadas de acuerdo a 163 definiciones preestablecidas, ${ }^{9}$ incluyendo complicaciones generales (CG) y relacionadas con procedimientos $(\mathrm{CP})$. Además fueron clasificadas como tempranas (CT) $(<72$ horas) y tardías ( $>72$ horas).

Las variables continuas fueron tratadas según su distribución y expresadas como media y desvío standard (DS) o mediana y rango intercuartil (RIC). La distribución de las variables categóricas se expresa como frecuencias y porcentajes. Las diferencias entre medias se estudiaron según test $\mathrm{T}$ de Student y, en caso de medianas, se utilizó el test U de Mann-Whitney. La asociación entre variables categóricas se estudió mediante el test de $\mathrm{Chi}^{2}$ o test de Fisher si la frecuencia esperada en alguna celda fuera menor a 5. La asociación entre variables predisponentes y resultados se expresó como odds ratio (OR) cuando se consideró apropiado. Las variables asociadas significativamente a la presencia de complicaciones tempranas ( $\mathrm{p}<$ 0.1) fueron incluidas en un análisis de regresión logística con selección manual. El modelo final propuesto fue testeado para los supuestos habituales: homocedasticidad, colinealidad e interacciones.

Los tests de hipótesis fueron realizados a dos colas, considerando un valor de $\mathrm{p}<0.05$ como estadísticamente significativo. El modelo final fue testeado en un set de validación mediante curva ROC.

\section{RESULTADOS}

Se reportaron 4521 complicaciones en 1883 pacientes (22\% de la muestra), mediana 1 , RIC 1-3, p/paciente (Tabla 1), distribuyéndose en 3586 CG (79\%) y 935 CP (21\%). Las CG se concentraron más en las primeras 72hs: CG vs CP: 69 vs 49\% (p < 0,001). Las CG más frecuentes fueron paro cardiorrespiratorio $(8,6 \%)$, neumonía asociada al ventilador (5,6\%), delirio (4,7\%) e hipertensión endocraneana (4\%) (Tabla 2). Excepto delirio, el resto de estas complicaciones fue significativamente más frecuente en no sobrevivientes.

Las CP más comunes fueron: inconvenientes en disposición de insumos (64\%) y en la derivación de pacientes (14\%). Este grupo de complicaciones se distribuyó por igual en ambos grupos (Tabla 3, Figuras 1 and 2).

La mortalidad global fue 5.5\%; en el grupo de CT fue $29.4 \%$, claramente superior al resto $(1.5 \%)$. La cantidad de complicaciones estuvo asociada significativamente a mortalidad ( $\mathrm{p}<0.001$, test U de Mann-Whitney) sin 
Tabla 1: Características basales

\begin{tabular}{llll}
\hline & $\begin{array}{l}\text { Complicaciones } \\
\text { tempranas NO }(n=7184)\end{array}$ & $\begin{array}{l}\text { Complicaciones } \\
\text { tempranas Si }(n=1198)\end{array}$ & $p$-value \\
\hline Edad (años) $^{*}$ & $22(11-35)$ & $29(20-43)$ & $<0.001^{\$}$ \\
ISS $^{*}$ & $5(4-9)$ & $16(9-25)$ & $<0.001^{\$}$ \\
NISS $^{*}$ & $6(4-11)$ & $18(9-34)$ & $<0.001^{\$}$ \\
Género femenino $^{\#}$ & $2026(28,2)$ & $5150(20,3)$ & $<0.001^{!}$ \\
GCS $^{\#}$ & $117(1,6)$ & $202(16,9)$ & $<0.001^{\S}$ \\
Lesiones por transporte $^{\S}$ & $2324(32,3)$ & $506(42,2)$ & $<0.001^{\S}$ \\
Shock al ingreso $^{\$}$ & $110(3,3)$ & $89(12,6)$ & $<0.001^{\S}$ \\
Trauma contuso $^{\$}$ & $5062(77)$ & $828(72,8)$ & $0.357^{\S}$ \\
Mortalidad $^{\#}$ & $108(1,5)$ & $353(29,5)$ & $<0.001^{!}$ \\
\hline
\end{tabular}

*Mediana (pc25-75); ${ }^{\#} \mathrm{n}(\%) ;{ }^{\$}$ Test U de Mann-Whitney; 'Test de Fisher; ${ }^{\S}$ Test Chi ${ }^{2}$

Tabla 2: Complicaciones generales

\begin{tabular}{|c|c|c|}
\hline & $\begin{array}{l}\text { Sobrevivientes } \\
(n=7921)\end{array}$ & $\begin{array}{l}\text { No } \\
\text { sobrevivientes } \\
(n=461)\end{array}$ \\
\hline Paro cardiorrespiratorio & $17(0,2)$ & $293(63,6)$ \\
\hline Neumonía asociada a AVM & $143(1,8)$ & $58(12,6)$ \\
\hline Delirio & $163(2,1)$ & $7(1,5)$ \\
\hline Hipertensión endocraneana & $63(0,8)$ & $79(17,1)$ \\
\hline Shock hemorrágico & $74(0,9)$ & $47(10,2)$ \\
\hline Ulcera por decúbito & $98(1,2)$ & $18(3,9)$ \\
\hline $\begin{array}{l}\text { Síndrome de distrés } \\
\text { respiratorio agudo (SDRA) }\end{array}$ & $47(0,6)$ & $55(11,9)$ \\
\hline Sepsis & $68(0,9)$ & $34(7,4)$ \\
\hline Shock séptico & $49(0,6)$ & $44(9,5)$ \\
\hline Neumonía intrahospitalaria & $75(0,9)$ & $15(3,3)$ \\
\hline Falla hemodinámica & $31(0,4)$ & $51(11,1)$ \\
\hline Infección urinaria & $69(0,9)$ & $11(2,4)$ \\
\hline Neumotórax & $52(0,7)$ & $27(5,9)$ \\
\hline Falla respiratoria & $46(0,6)$ & $26(5,6)$ \\
\hline Síndrome convulsivo & $56(0,7)$ & $14(3)$ \\
\hline Muerte encefálica & $1(0)$ & $67(14,5)$ \\
\hline Atelectasia & $49(0,6)$ & $18(3,9)$ \\
\hline $\begin{array}{l}\text { Infección de piel y partes } \\
\text { blandas }\end{array}$ & $58(0,7)$ & $5(1,1)$ \\
\hline Infección de herida quirúrgica & $55(0,7)$ & $6(1,3)$ \\
\hline Infección asociada a catéter & $50(0,6)$ & $10(2,2)$ \\
\hline Neumonía aspirativa & $44(0,6)$ & $15(3,3)$ \\
\hline Insuficiencia renal aguda & $28(0,4)$ & $30(6,5)$ \\
\hline Sindrome febril prolongado & $44(0,6)$ & $10(2,2)$ \\
\hline Otras complicaciones & $48(0,6)$ & $6(1,3)$ \\
\hline Arritmia & $23(0,3)$ & $26(5,6)$ \\
\hline Hipotensión & $32(0,4)$ & $15(3,3)$ \\
\hline Infección endovascular & $35(0,4)$ & $10(2,2)$ \\
\hline Hipertensión arterial & $33(0,4)$ & $11(2,4)$ \\
\hline Meningitis & $27(0,3)$ & $15(3,3)$ \\
\hline Extubación no programada & $37(0,5)$ & $2(0,4)$ \\
\hline Rabdomiolisis & $27(0,3)$ & $9(2)$ \\
\hline Coagulopatía & $12(0,2)$ & $22(4,8)$ \\
\hline Derrame pleural & $23(0,3)$ & $10(2,2)$ \\
\hline Flebitis & $29(0,4)$ & $4(0,9)$ \\
\hline
\end{tabular}

encontrar diferencias entre géneros (Figuras 1 y 2). El OR para mortalidad en el grupo de CT fue 27,4 (IC 95\% 22-34) quintuplicando el de complicaciones totales.

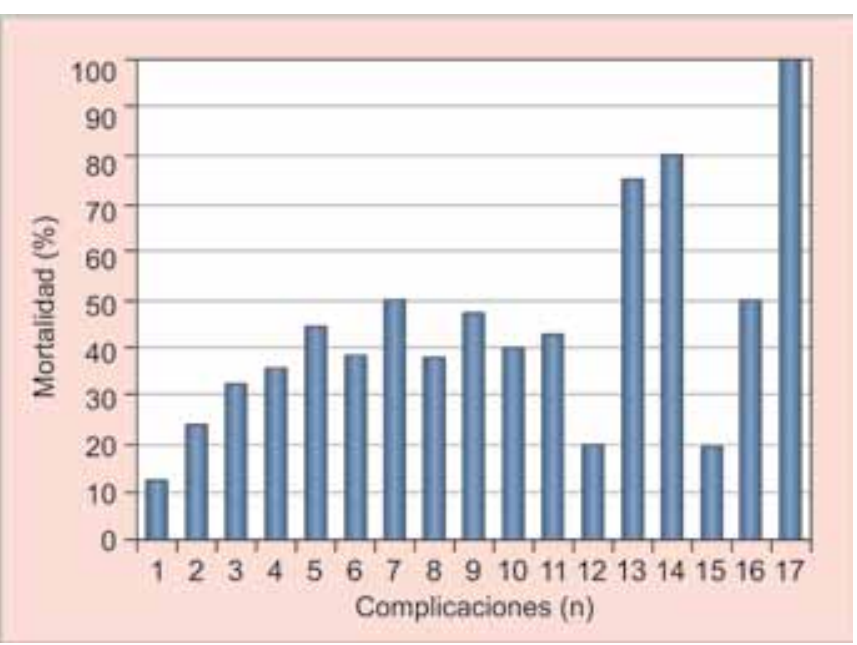

Figura 1: Cantidad de complicaciones y mortalidad

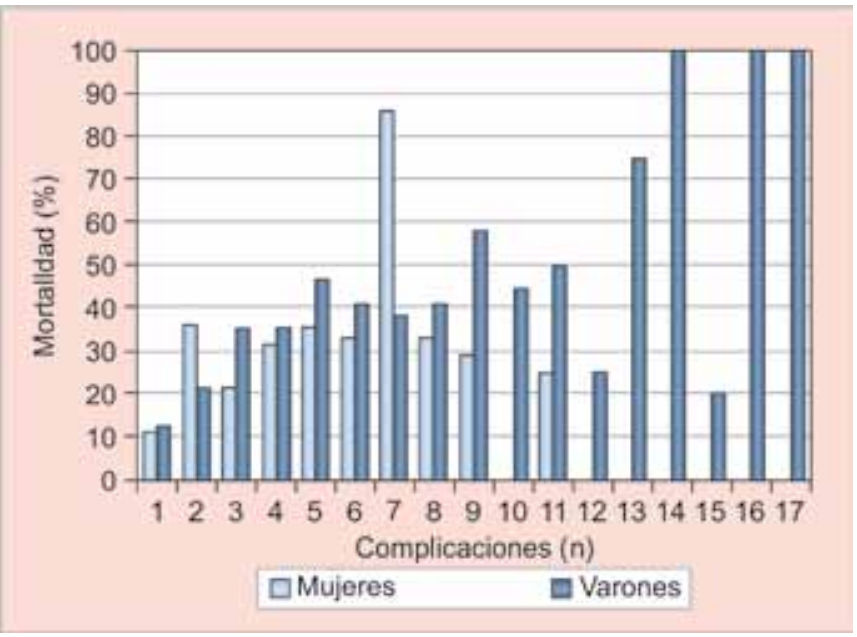

Figura 2: Cantidad de complicaciones y mortalidad según género

En la regresión logística, luego de eliminar interacciones y variables no significativamente asociadas al resultado, el modelo integrado por edad $<20$ años, ISS $>8$, shock al ingreso y GCS $<9$ se asoció a un OR de 11.5 (IC95\% 8.8-15.0) de presentar CT (área bajo ROC 0,75) (Figura 3).

\section{DISCUSIÓN}

Este es el primer reporte de complicaciones surgido de un registro de trauma en la República Argentina. Como 
Tabla 3: Complicaciones asociadas a procedimientos

\begin{tabular}{lll}
\hline & $\begin{array}{l}\text { Sobrevivientes } \\
(n=7921)\end{array}$ & $\begin{array}{l}\text { No. sobrevivientes } \\
(n=461)\end{array}$ \\
\hline Inconvenientes administrativos/institucionales en la disposición de insumos & $592(7,5)$ & $6(1,3)$ \\
Inconvenientes administrativos/institucionales en la derivación del paciente & $124(1,6)$ & $7(1,5)$ \\
Inconvenientes administrativos/institucionales en la obtención de estudios diagnósticos & $30(0,4)$ & $3(0,7)$ \\
Inconvenientes de técnica durante el o los procedimientos de diagnóstico, monitoreo o & $14(0,2)$ & $6(1,3)$ \\
terapéuticos & $16(0,2)$ & $(0)$ \\
Inconvenientes en la realización de la consulta médica especializada interinstitucional & $12(0,2)$ & $2(0,4)$ \\
Inconvenientes en la realización de la consulta médica especializada & $4(0,1)$ & $2(0,4)$ \\
Inconvenientes en la obtención de acceso intravenoso/intraóseo & $5(0,1)$ & $(0)$ \\
Inconvenientes en la disponibilidad del equipo asistencial en el Departamento de & $2(0)$ & $1(0,2)$ \\
Emergencias & & \\
Inconvenientes en el diagnóstico & &
\end{tabular}

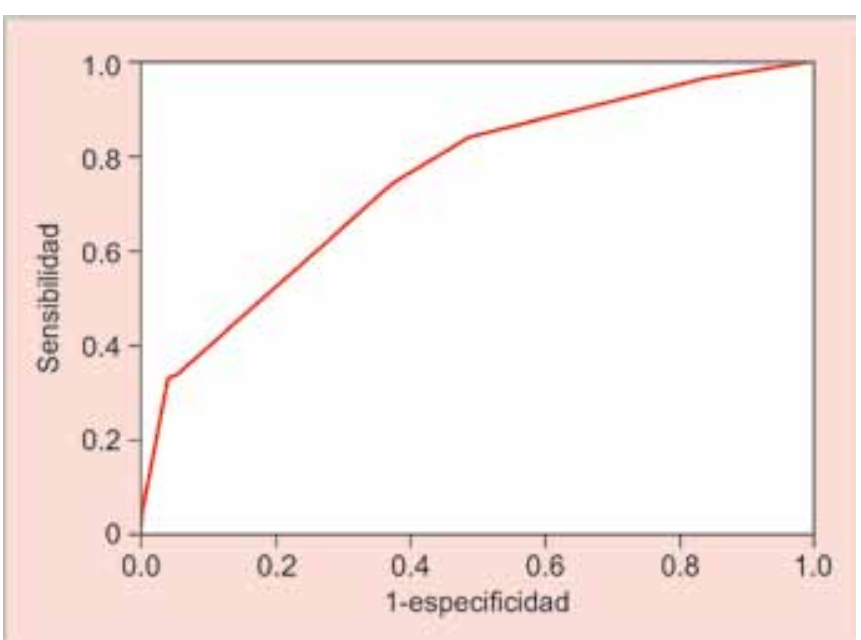

Figura 3: Curva ROC para la identificación de los pacientes con CT en base al modelo propuesto

fuera expresado anteriormente, el RT de la Fundación Trauma es una intervención dependiente de una organización no gubernamental e independiente de la administración estatal, lo cual brinda una situación preferencial al momento de registrar complicaciones del tratamiento. Consideramos que, si bien el mismo sirve para conocer el perfil de complicaciones ocurridas en ocho hospitales que asisten pacientes traumatizados de acuerdo a una metodología sólida y homogénea a través de las instituciones, también surgen algunos atenuantes de las conclusiones extraíbles que deberían considerarse: dado que no existe otro método que lleve a cabo este control, existe la posibilidad de estar incurriendo en subregistro de eventos, aspecto que no puede precisarse. Por otro lado, en esta primera etapa el RT registró las complicaciones de acuerdo a su constancia en la historia clínica de los pacientes, lo que incorpora también la heterogeneidad interinstitucional en relación a criterios diagnósticos.

Por otra parte, a nivel mundial no está claro aún cuáles complicaciones son más significativas para usar como indicadores de performance del tratamiento de pacientes traumatizados. ${ }^{10}$ Esto incrementa la heterogeneidad y dificulta la comparación entre muestras de diferentes centros y regiones.

En este estudio, a diferencia de otros, no se encontraron diferencias significativas en las complicaciones que dependieran del género. ${ }^{11-13}$ Algunas causas para justificar este comportamiento podrían ser el bajo tamaño muestral (especialmente en el género femenino) y la presencia de subregistro, que podría sesgar alguna tendencia, sin descartar, por supuesto, que esta sea una característica propia de la asistencia en este medio. Se estima poder revisar este aspecto a la luz de la incorporación de una muestra más representativa. Como dato llamativo, se destaca la elevada tasa de reporte de complicaciones administrativas, ubicadas en los tres primeros lugares en incidencia de las complicaciones asociadas a procedimientos. Estas complicaciones, a diferencia de las generales, no mostraron asociación con los resultados.

\section{CONCLUSIONES}

En una muestra de pacientes traumatizados internados obtenida de cuatro años en ocho hospitales argentinos, se observó un perfil específico de paciente (edad $<20$ años, ISS $>8$, shock al ingreso y GCS $<$ 9) con mayor riesgo de sufrir CT, lo que puede ayudar en las estrategias de MCA.

\section{REFERENCIAS}

1. Dirección de Estadísticas e Información en Salud. Boletín nro. 144. Disponible en http://www.deis.gov.ar/publicaciones/ Archivos/Boletin144.pdf.

2. Mann N, Mullins R, MacKenzie E, Jurkovich G, Mock C. Systematic review of published evidence regarding trauma system effectiveness. J Trauma 1999;47(3 suppl):S25-33.

3. MacKenzie EJ, Rivara FP, Jurkovich GJ, Nathens AB, Frey KP et al. A national evaluation of the effect of trauma-center care on mortality. N Engl J Med 2006 Jan 26;354(4):366-378.

4. Jurkovich GJ, Mock C. Systematic review of trauma system effectiveness based on registry comparisons. J Trauma 1999; 47(3 Suppl):S46-55. 
5. Mullins RJ, Mann NC. Population-based research assessing the effectiveness of trauma systems. J Trauma 1999;47:S59-66.

6. Glance LG, et al. Outcomes of adult trauma patients admitted to trauma centers in Pennsylvania, 2000-2009. Archives of Surgery 2012 Aug;147(8):732-737.

7. Mackenzie EJ, Rivara FP, Jurkovich GJ, Nathens AB, Frey KP, et al. The national study on costs and outcomes of trauma. J Trauma 2007;63(6 Suppl):S54-S67.

8. Mock C, et al. Guidelines for trauma quality improvement programmes. Geneva: WHO; 2009.

9. Hoyt DB, Hollingsworth-Fridlund P, Fortlage D, Davis JW, Mackersie RC. An evaluation of provider related and disease related morbilidty in a Level I University Trauma Service: directions for Quality Improvement. J Trauma 1992 Oct; 33(4):586-601.
10. Moore L, Stelfox HT, Turgeon AF. Complication rates as a trauma care performance indicator: a systematic review. Crit Care 2012;16:R195.

11. Mondello S, Cantrell A, Italiano D, Fodale V, Mondello P, Ang D. Complications of Trauma Patients Admitted to the ICU in Level I Academic Trauma Centers in the United States. Biomed Res Int 2014;Art ID 473419.

12. Napolitano LM, Greco ME, Rodriguez A, Kufera JA, West RS, Scalea TM. Gender differences in adverse outcomes after blunt trauma. J Trauma 2001;50(2):274-280.

13. Magnotti LJ, Fischer PE, Zarzaur BL, Fabian TC, Croce MA. Impact of gender on outcomes after blunt injury: a definitive analysis of more than 36, 000 trauma patients. J Am Coll Surg 2008;206(5):984-991. 


\section{Perfil de Complicaciones Tempranas en la Atención del Paciente Traumatizado}

En este interesante estudio, Monteverde y sus colegas trataron de delimitar la incidencia y tipo de complicaciones que se producen en los pacientes con trauma y determinar los distintos perfiles de riesgo asociados a complicaciones tempranas. Las complicaciones tempranas fueron definidas como las complicaciones que ocurren $<72$ horas. Los autores llevaron a cabo un estudio observacional retrospectivo utilizando datos sin identificación a partir de ocho hospitales de la provincia de Buenos Aires en Argentina que someten los datos al Registro de Trauma de la Fundación de Trauma. El estudio se llevó a cabo durante un período de 3,5 años a partir de Enero de 2010 a Julio de 2014. De los 10.772 pacientes ingresados durante el período de estudio, 8.382 fueron incluidos en el estudio. De los 1.883 o 22 \% fueron reportados como tener complicaciones. 1.198 o el 64\% de los pacientes sufrió complicaciones en las primeras 72 horas.

Los autores reportaron un total de 4521 complicaciones. $79 \%$ de las complicaciones se atribuyeron a complicaciones generales (clínicas) y se incluye la paro cardiorrespiratorio (8,6\%), neumonía asociada a ventilación mecánica (5,6\%) , el delirio (4,7\%) y la hipertensión intracraneal (4\%). Los autores también reportaron una mortalidad total del 5,5\%. De los que murieron, 353 o el $77 \%$ se produjo entre 23 y 72 horas resultando en casi un 30\% la mortalidad temprana en comparación con el 1,5\% de mortalidad (> 72 horas).

Para determinar los factores de riesgo para las complicaciones tempranas, los autores llevaron a cabo un modelo de regresión logística donde la edad < 20 años, ISS > 8, shock al ingreso, y GCS < 9 se asociaron con un OR de 11,5 de tener complicaciones tempranas.

Animo a la autora para dar una mejor descripción de las características de los hospitales que contribuyen al registro de datos, si las tasas de complicaciones y mortalidad se distribuyeron por igual en todos los ocho hospitales. Además, las tendencias en el tiempo, en términos de mortalidad y morbilidad también serían un factor importante para delinear una vez que se iniciaron los esfuerzos de mejora de calidad. También me gustaría animar a los autores a incluir el tipo de esfuerzos de mejora de la calidad que pueden ser incluidos sobre todo en la fase inicial $<72$ horas para tener un impacto en las complicaciones identificadas.

Por último y lo más importante, me gustaría felicitar a los autores en el uso de un registro regional de trauma para delinear las características importantes y necesarias en los pacientes con trauma de la provincia de Buenos Aires. Sin esos datos, las estrategias de prevención e intervención, junto con los esfuerzos de mejora de la calidad no pueden ser llevadas a cabo. Lesiones relacionadas con el trauma siguen siendo la principal causa de muerte cada año en Argentina, Estados Unidos y todo el mundo. Importantes esfuerzos y estudios se han centrado en la mortalidad por lesiones, que no refleja adecuadamente la verdadera carga de la enfermedad conocida como trauma en la sociedad en su conjunto. La morbilidad de lesiones sin embargo y su impacto en términos de resultados de salud y el costo es un factor vital para delinear y mejorar la prestación de la asistencia sanitaria. La delimitación de las complicaciones atribuibles a las tasas de mortalidad y morbilidad se ha convertido en un foco esencial de los esfuerzos de mejora de calidad, especialmente para la evaluación comparativa de los esfuerzos y la asignación de recursos adecuados. La escasez de datos de calidad sin embargo, en muchos países se opone a este tipo de análisis importante. Esto pone en perspectiva la importancia de este estudio.

Los datos de mortalidad de 5,5\% observado es comparable al reportados estadísticas nacionales y de la OMS. Sin embargo, la atención a la complicación temprana y los factores de riesgo asociados delineadas en este estudio, se presta para los esfuerzos futuros de mejora del rendimiento enfocado a reducir la probabilidad de resultados pobres. Tales esfuerzos no podían ser enfocados antes de la iniciación y uso de registros de datos como se indica en este estudio.

Felicito a los autores y espero con ansias más estudios de la Fundación de Trauma.

Michel Aboutanos

Professor and Chief

Department of Surgery

Acute Care Surgical Services

VCU Medical Center, Richmond, Virginia, USA 


\section{INVITED COMMENTARY}

\section{Early Complications Profile in Traumatized Patients}

In this interesting study, Monteverde and colleagues sought to delineate the incidence and type of complications that occur in trauma patients and to determine the various risk profiles associated with early complications. Early complications were defined as complications occurring in $<72$ hours. The authors carried out a retrospective observational study using deidentified data from eight hospitals in the province of Buenos Aires in Argentina who submit data to the Trauma Registry of the Trauma Foundation. The study was carried out over a period of 3.5 years from January 2010 to July 2014. Of the 10,772 patient admitted during the study period, 8382 were included in the study. Of those $1883(22 \%)$ were reported as having complications. Around $1198(64 \%)$ of the patients sustained complications within the first 72 hours.

The authors reported a total of 4521 complications. Around $79 \%$ of the complications were attributed to general (clinical) complications and included cardiopulmonary arrest (8.6\%), ventilator-associated pneumonia (5.6\%), delirium $(4.7 \%)$ and intracranial hypertension ( $4 \%$ ). The authors also reported a total mortality of $5.5 \%$. Of those who died, 353 or $77 \%$ occurred between 23 and 72 hours resulting in almost $30 \%$ early mortality compared to $1.5 \%$ mortality ( $>72$ hours).

To determine risk factors for early complications, the authors carried out a logistic regression model where age $<20$ years, ISS $>8$, shock at admission, and GCS $<9$ were associated with an OR of 11.5 of having early complications.

I encourage the authors to give a better description of the characteristics of the hospitals contributing to the data registry, whether the complication and mortality rates were equally distributed across all eight hospitals. Additionally, the trends over time, in terms of mortality and morbidity would also be an important factor to delineate once quality improvement efforts are initiated. I would also encourage the authors to include the type of quality improvement efforts that could be included especially in the early phase $<72$ hours to have an impact on the identified complications.

Finally and most importantly, I would like to congratulate the authors on the use of a regional trauma registry to delineate important and needed characteristics on trauma patients from the province of Buenos Aires. Without such data, prevention and intervention strategies along with quality improvement efforts cannot be carried out. Traumarelated injuries remain a leading cause of death each year in Argentina, United States and World Wide. Significant efforts and studies have focused on injury mortality, which does not adequately reflect the true burden of disease known as trauma on the society as a whole. Injury morbidity however and its impact in terms of healthcare outcomes and cost is a vital factor to delineate and improve healthcare delivery. The delineation of attributable complications to both mortality rates and morbidity rates has become an essential focus of quality improvement efforts especially for benchmarking efforts and appropriate resource allocation. ${ }^{2-4}$ The paucity of quality data however in many countries preclude such important analysis. This puts in perspective the importance of this current study.

The noted 5.5\% mortality data is comparable to reported national and WHO statistics. However, the attention to early complication and the associated risk factors delineated in this study, lends for future focused performance improvement efforts to reduce the probability of poor outcomes. ${ }^{1-6}$ Such efforts could not be focused prior to the initiation and use of data registries as noted in this study.

I congratulate the authors and look forward to further studies from the Trauma Foundation.

\section{REFERENCES}

1. Ingraham AM, et al. The attributable mortality and length of stay of trauma-related complications: a matched cohort study. Annals Surg 2010;252(2):358-362.

2. Dimick JB, et al. Complications and costs after high-risk surgery: where should we focus quality improvement initiatives? J Am Coll Surg 196(5):671-678.

3. Heron M, et al. National Vital Statistics Reports. Atlanta (GA): Centers for Disease Control and Prevention; 2009. Accessed on: May 2015.

4. Glance LG, et al. Outcomes of adult trauma patients to trauma centers in Pennsylvania, 2000-2009. Arch Surg 2012;147(8):732-737.

5. MacKenzie EJ, et al. The national study on costs and outcomes of trauma. J Trauma 2007;63(6 Suppl):S54-S67.

6. Moore L, et al. Complication rates as a performance indicator trauma care: a systematic review. Crit Care 2012 Oct 16;16(5):R195.

Michel Aboutanos

Professor and Chief

Department of Surgery

Acute Care Surgical Services

VCU Medical Center, Richmond, Virginia, USA 